\title{
Age-dependent association of white matter abnormality with cognition after TIA or minor stroke
}

Giovanna Zamboni, MD, DPhil, Ludovica Griffanti, PhD, Sara Mazzucco, MD, PhD, Sarah T. Pendlebury, MD, DPhil, FRCP, and Peter M. Rothwell, MD, PhD, FMedSci

Neurology ${ }^{\circledR}$ 2019;93:e272-e282. doi:10.1212/WNL.0000000000007772

\author{
Correspondence \\ Dr. Zamboni \\ Giovanna.zamboni@ \\ unimore.it
}

\begin{abstract}
Objective

To investigate if the association between MRI-detectable white matter hyperintensity (WMH) and cognitive status reported in previous studies persists at older ages ( $>80$ years), when some white matter abnormality is almost universally reported in clinical practice.
\end{abstract}

\section{Methods}

Consecutive eligible patients from a population-based cohort of all TIA/nondisabling stroke (Oxford Vascular Study) underwent multimodal MRI, including fluid-attenuated inversion recovery and diffusion-weighted imaging, allowing automated measurement of WMH volume, mean diffusivity (MD), and fractional anisotropy (FA) in normal-appearing white matter using FSL tools. These measures were related to cognitive status (Montreal Cognitive Assessment) at age $\leq 80$ vs $>80$ years.

\section{Results}

Of 566 patients (mean [range] age 66.7 [20-102] years), 107 were aged $>80$ years. WMH volumes and $\mathrm{MD} / \mathrm{FA}$ were strongly associated with cognitive status in patients aged $\leq 80$ years (all $p<0.001$ for $\mathrm{WMH}, \mathrm{MD}$, and FA) but not in patients aged $>80$ years (not significant for $\mathrm{WMH}, \mathrm{MD}$, and FA), with age interactions for $\mathrm{WMH}$ volume $\left(p_{\text {interaction }}=0.016\right)$ and $\mathrm{MD}$ $\left(p_{\text {interaction }}=0.037\right)$. Voxel-wise analyses also showed that lower Montreal Cognitive Assessment scores were associated with frontal WMH in patients $\leq 80$ years, but not $>80$ years.

\section{Conclusion}

MRI markers of white matter damage are strongly related to cognition in patients with TIA/ minor stroke at younger ages, but not at age $>80$ years. Clinicians and patients should not overinterpret the significance of these abnormalities at older ages. 


\section{Glossary}

ANOVA $=$ analysis of variance; BET $=$ brain extraction tool; BIANCA $=$ brain intensity abnormality classification algorithm; CI = confidence interval; DTI $=$ diffusion tensor imaging; FA = fractional anisotropy; FLAIR = fluid-attenuated inversion recovery; FNIRT = FMRIB's nonlinear image registration tool; FOV = field of view; GRAPPA = generalized autocalibrating partial parallel acquisition; $\mathbf{M D}=$ mean diffusivity; MildCI = mild cognitive impairment; $\mathbf{M N I}=$ Montreal Neurological Institute; $\mathbf{M o C A}=$ Montreal Cognitive Assessment; NAWM = normal-appearing white matter; NoCI $=$ no cognitive impairment; OR = odds ratio; OXVASC = Oxford Vascular Study; SevereCI = severe cognitive impairment; TE = echo time; TI = inversion time; $\mathbf{T R}=$ repetition time; $\mathbf{W M H}=$ white matter hyperintensity.

White matter hyperintensity (WMH) of presumed vascular origin detectable on MRI is associated with cognitive impairment and dementia. ${ }^{1,2}$ Associations between cognitive scores and measures of WMH load have been shown in elderly individuals without dementia, ${ }^{3-6}$ patients with manifested arterial disease, ${ }^{7}$ and patients with TIA or minor stroke. ${ }^{8}$ Measures of white matter microstructural integrity estimated using diffusion tensor imaging (DTI), such as fractional anisotropy (FA) and mean diffusivity (MD), are also associated with cognitive deficits in elderly individuals without dementia ${ }^{9}$ including those with small vessel disease. ${ }^{10,11}$ Importantly, it has been shown that these DTI measures are abnormal not only in WMH regions, but also in the surrounding normal-appearing white matter (NAWM), and that the level of DTI-detected deterioration of NAWM is associated with age and WMH burden in cognitively healthy adults, ${ }^{12,13}$ including those older than $90,{ }^{14}$ as well as poststroke patients. ${ }^{15,16}$ These findings suggest that DTI modifications precede the occurrence of $\mathrm{WMH}$ and better capture the true extent of pathophysiologic changes underlying global white matter. ${ }^{17,18}$

The prevalence of WMH increases with age, particularly after age $80,{ }^{19,20}$ and DTI estimates of white matter integrity also sharply deteriorate with age. ${ }^{21}$ However, there are few data on the agespecific association between MRI-detectable white matter damage and cognition, with most studies reporting associations pooled across a broad range of ages (e.g., $\geq 50,{ }^{7}>60,{ }^{3}$ or $>65^{22}$ ). Yet, some studies suggest that the association may attenuate at age $>80,{ }^{23,24}$ although, to our knowledge, no studies have directly compared the association in older vs younger adults.

Establishing the relevance of WMH to cognition in very old patients is increasingly important because individuals over 80 years of age represent the most rapidly growing segment of the population, ${ }^{25}$ with the greatest concern about risk of dementia. ${ }^{26-28}$ Furthermore, MRI is now very frequently performed as first-line brain imaging for a wide range of neurologic symptoms, such that some WMH are almost always reported in older patients. ${ }^{19,29}$ The most common indication for such imaging in routine practice is after TIA and stroke, and patients frequently have evidence of small vessel disease, inevitably raising concern about vascular cognitive impairment. ${ }^{30-32} \mathrm{We}$ therefore studied MRI markers of white matter damage and cognitive status in a population-based cohort of patients with TIA or minor stroke, comparing those aged $\leq 80$ vs $>80$ years.
We also explored with voxel-wise analyses what WMH location is more strongly associated with cognitive impairment and whether there would be differences between age groups.

\section{Methods}

\section{Study population}

Consecutive patients were recruited between March 2012 and June 2016 from the Oxford Vascular Study (OXVASC), a prospective cohort study of all acute vascular events in a defined population of 92,000 residents registered with 100 primary care physicians in Oxfordshire, and the only population-based study of all vascular disease that does not exclude very old patients. After a suspected nondisabling cerebrovascular event (NIH Stroke Scale score <4), OXVASC participants undergo brain MRI, detailed clinical characterization, and cognitive assessment, with face-to-face follow-up at $1,3,6,12,24$, and 60 months. In order to avoid any selection bias, particularly against older patients, patients with a previous TIA or minor stroke prior to the imaging study period were included. Exclusion criteria specific for the purposes of the present imaging study were (1) MRI contraindication or known claustrophobia; (2) intracranial spaceoccupying lesion; (3) intracranial hemorrhage; (4) brain defect due to previous neurosurgery or developmental anomalies; (4) large chronic, subacute, or acute infarcts (i.e., $>2.5 \mathrm{~cm}$ on T1-weighted, T2-weighted, or diffusionweighted imaging sequences); (5) significant movement artefacts that would impair registration; (6) inability to perform cognitive testing (i.e., due to language barriers).

\section{Standard protocol approvals, registrations, and patient consents}

Written informed consent was obtained from all participants. OXVASC was approved by the local ethics committee (Research Ethics Committee reference number: 05/Q1604/70).

\section{Data availability}

Requests for data from the OXVASC Study will be considered by P.M.R. in line with data protection laws. The general policy is that as long as the proposed use of the data is scientifically valid and as long as ethics approval permits, suitably anonymized data can be shared with other researchers. 


\section{Cognitive status}

Participants were divided into 3 groups according to their Montreal Cognitive Assessment (MoCA) scores, which has been shown to be sensitive to detect vascular cognitive impairment ${ }^{33-35}$ : no cognitive impairment (NoCI, MoCA $\geq 26$ ), mild cognitive impairment (MildCI, $20<\mathrm{MoCA}<26$ ), or severe cognitive impairment (SevereCI, MoCA $\leq 20$ ). These cutoffs were chosen on the basis of previous work showing that the MoCA has high sensitivity in identifying poststroke patients with mild but also severe/multidomain cognitive impairment. ${ }^{34,36}$ For the purpose of the present study, we used MoCA scores from the 1-month follow-up as these better reflect the cognitive status independent from transient cognitive variations related to the minor cerebrovascular event. ${ }^{37}$

\section{Imaging acquisition}

All images were acquired on a 3T Verio (Orem, UT) MRI scanner. The imaging protocol used until December 2014 included fluid-attenuated inversion recovery (FLAIR) (repetition time $[\mathrm{TR}] /$ echo time $[\mathrm{TE}] /$ inversion time $[\mathrm{TI}]$ 9,000/ $94.0 / 2,500 \mathrm{~ms}$, flip angle $150^{\circ}$, field of view [FOV] $200 \mathrm{~mm}$, voxel size $0.8 \times 0.8 \times 5 \mathrm{~mm}$ with $1.5 \mathrm{~mm}$ interslice gap), postgadolinium T1-weighted imaging (TR/TE/TI 1,250/4.63/ $900 \mathrm{~ms}$, flip angle $16^{\circ}$, FOV $220 \mathrm{~mm}$, voxel size $1.1 \times 1.1 \times$ $3 \mathrm{~mm}$ with $1.5 \mathrm{~mm}$ interslice gap), and diffusion-weighted imaging (TR/TE 4,000/106 ms, generalized autocalibrating partial parallel acquisition [GRAPPA] factor 2, FOV $230 \mathrm{~mm}$, voxel size $1.8 \times 1.8 \times 4 \mathrm{~mm}$ with $1.2 \mathrm{~mm}$ interslice gap, 12 directions, $\mathrm{b}$ value $1,000 \mathrm{~s} / \mathrm{mm}^{2}$ ).

The protocol used from January 2015 included highresolution T1 (TR/TE/TI 2,000/1.94/880 ms, flip angle $8^{\circ}$, FOV $256 \mathrm{~mm}$, voxel size $1 \times 1 \times 1 \mathrm{~mm}$ ), FLAIR (TR/TE/TI $9,000 / 88 / 2,500 \mathrm{~ms}$, flip angle $150^{\circ}$, FOV $192 \mathrm{~mm}$, voxel size 1 $\times 1 \times 3 \mathrm{~mm})$, and diffusion-weighted imaging $(\mathrm{TR} / \mathrm{TE}=$ $8,000 / 86 \mathrm{~ms}$, GRAPPA factor 2, flip angle $16^{\circ}$, FOV $192 \mathrm{~mm}$, voxel size $2 \times 2 \times 2 \mathrm{~mm}, 32$ directions, b value $1,500 \mathrm{~s} / \mathrm{mm}^{2}$ ).

Measures of white matter damage (WMH volumes, MD and FA in NAWM) obtained from the second protocol were standardized on values obtained from the first protocol to allow statistical analyses across the whole sample. In addition, protocol type was added as covariate of no interest on univariate and voxel-wise analyses.

Presence/absence of lacunar infarcts was rated by stroke neurologists and neuroradiologists who were blind to the cognitive scores. Lacunar infarcts were defined as hypointense lesions on T1 imaging with corresponding hyperintense lesion on FLAIR images with a diameter $<15 \mathrm{~mm}$.

\section{WMH measurement}

WMHs were automatically segmented on FLAIR images with brain intensity abnormality classification algorithm (BIANCA), a fully automated, supervised method for WMH detection, which gives the probability per voxel of being
$\mathrm{WMH}^{38}$ The total WMH volume was calculated from the voxels exceeding a probability of 0.9 (which gave the highest accuracy on this dataset, as tested in our previous work ${ }^{38}$ ) of being WMH and located within a white matter mask. Obtained values were adjusted for the total brain and ventricles volume (i.e., the sum of the volumes of gray matter, white matter, and ventricles) calculated from the brainextracted images using FSL's brain extraction tool (BET) ${ }^{39}$ and log transformed, ${ }^{40}$ as a proxy for intracranial volume that could be obtained from the available FLAIR images.

For voxel-wise analyses, the thresholded and masked WMH maps were binarized and transformed into Montreal Neurological Institute (MNI) standard space, applying the nonlinear registration (FMRIB's nonlinear image registration tool $[\text { FNIRT] })^{41}$ calculated from FLAIR to MNI (via highresolution $\mathrm{T} 1$, if available). We further thresholded the transformed maps at 0.5 , binarized them, and applied spatial smoothing of full width at half maximum $=6 \mathrm{~mm}$ to compensate for registration errors (the size of the smoothing kernel was empirically decided to be the same as the maximum voxel dimension). The resulting maps were entered into voxel-wise WMH statistical analyses.

WMH were also visually rated on the Fazekas WMH scale allowing categorical measurement of periventricular and deep $\mathrm{WMH}$ in grades from 0 (absent) to 3 (severe). ${ }^{42}$

\section{Measurements of microstructural white matter integrity in NAWM}

Diffusion-weighted images were first corrected for head motion and eddy currents. DTI was then performed to create MD and FA maps by fitting a tensor model to the diffusionweighted images using FMRIB's Diffusion Toolbox. ${ }^{43}$ Images were brain-extracted using FSL's BET. All participants' FA maps were then nonlinearly registered to a common diffusion space (FMRIB58_FA, an FA template in MNI space) using FNIRT and the same transformation was applied to MD data. For each participant, we calculated the linear transformation from FLAIR to diffusion data ( $b=0$ image used as reference) and combined it with the nonlinear transformation from diffusion data to the common space calculated before. The resulting transformation was then applied to WMH maps to register them from FLAIR to diffusion common space. MD and FA values from the NAWM were calculated as average from voxels outside the WMH map, within a mask including all the main white matter tracts in the JHU-ICBM DTI atlas (figure 1). The evaluation of DTI-derived measures was restricted to the main white matter tracts and not performed in the whole NAWM in order to focus on the tracts that are more consistent across participants. This also allows compensating for possible registration errors occurring in the rest of the white matter. In addition, to exclude the possibility of a bias in the results due to a systematic difference in registration quality across age groups, we calculated a cost metric (root mean square difference) between each participant's FA image registered to the template and the template itself and 


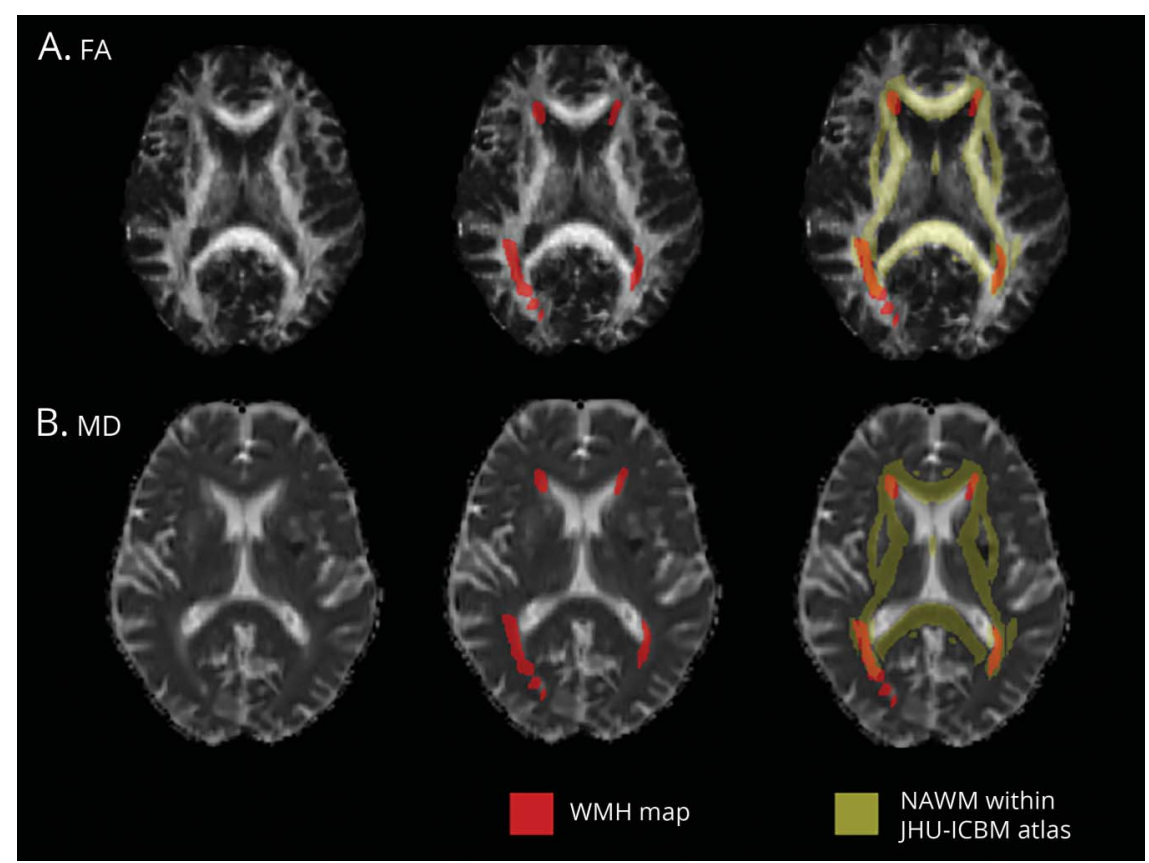

Examples of (A) fractional anisotropy (FA) and (B) mean diffusivity (MD) maps registered in Montreal Neurological Institute space from a single participant. Average FA and MD value within (NAWM) was calculated from the regions shown in yellow, that is, within a mask including all the main white matter tracts from the JHU-ICBM DTI atlas and excluding regions of white matter hyperintensity (WMH) obtained with brain intensity abnormality classification algorithm (red). verified that it was not significantly different between age groups (estimated root mean square difference $=0.0420 \pm$ $0.0039 ;<80$ years only $=0.0418 \pm 0.0039 ;>80$ years only $=$ $0.0426 \pm 0.0041)$.

\section{Statistical analyses}

Patients were grouped by age $\leq 80$ vs $>80$ years and cognitive status (NoCI, MildCI, SevereCI, respectively) as defined above. Comparisons between age groups were performed with Mann-Whitney or independent $t$ test, as appropriate, for continuous variables, and $\chi^{2}$ tests for dichotomous variables using SPSS version 22.0 (SPSS Inc., Chicago, IL). Age-related differences in the associations between MRI markers of white matter damage and cognitive status were studied with $2 \times 3$ factorial analyses of variance (ANOVAs). Results were considered significant at $p<0.05$.

Sensitivity analyses were conducted to control for the effects of potential confounders. These analyses included MRI protocol, sex, years of education, head size, presence of lacunes, and number of vascular risks factors (i.e., the sum of hypertension, diabetes mellitus, atrial fibrillation, hyperlipidemia, and smoking). Furthermore, to account for differences in sample size between age groups, the ANOVA was repeated using 4 random subsamples of young participants stratified on cognitive status to match the original large sample.

\section{Voxel-wise analysis of WMH}

We performed the same $2 \times 3$ factorial ANOVA at the voxel level on the maps of WMH obtained with BIANCA to study the location in the brain of age-by-cognitive status interactions. We then performed correlational voxel-wise analysis on the same maps to test the association between higher probability of having $\mathrm{WMH}$ and lower MoCA score in patients aged $\leq 80$ and in patients aged $>80$, separately for the 2 groups.

All the statistical analyses were performed with nonparametric permutation tests using the randomise tool in FSL, ${ }^{44}$ with protocol as nuisance covariate, and restricted to a white matter mask. Results were considered significant at $p<0.05$ fully corrected for multiple comparisons using family-wise error correction at the voxel level. ${ }^{44}$

\section{Results}

Among 570 consecutive eligible patients, 4 were excluded due to subsequently diagnosed WMH mimics (multiple sclerosis) and known other causes of dementia (cerebral autosomal dominant arteriopathy with subcortical infarcts and leukoencephalopathy and Alzheimer disease). Table 1 reports the characteristics of the 566 patients included in the WMH analyses, also divided by age groups (i.e., $\leq 80$ and $>80$ ). Diffusion-weighted MRI, allowing measurement of FA and $\mathrm{MD}$, were acquired from a subsample of 498 consecutive participants (88\%), as this sequence was not initially included in the protocol. Sensitivity analyses on WMH volumes (available on 566 participants) were repeated in the subsample of 498 participants and gave similar results.

\section{Age-related associations between WMH volume and cognitive status}

A $2 \times 3$ factorial ANOVA on WMH volumes showed a main effect of age $(F=34.95, p<0.001)$, a main effect of cognition 
Table 1 Clinical and imaging features of total sample and age groups

\begin{tabular}{|c|c|c|c|c|}
\hline Study & Total & Age $\leq 80$ y & Age $>80 y$ & $p$ Value \\
\hline $\mathbf{N}$ & 566 & 459 & 107 & \\
\hline Age, $y$, mean \pm SD & $66.7 \pm 14.3$ & $62.5 \pm 12.5$ & $84.7 \pm 3.7$ & \\
\hline Age range, y & $20-102$ & $20-80$ & $81-102$ & \\
\hline Age, y, median (IQR) & $69(20.5)$ & 65 (17.9) & $83.8(4.9)$ & \\
\hline Female sex, $n(\%)$ & $280(49.5)$ & $228(49.7)$ & $52(48.6)$ & 0.841 \\
\hline TIA, other minor stroke, $n$ (\%) & $408(72.1)$ & $336(73.4)$ & $72(67.9)$ & 0.259 \\
\hline Years of education, mean \pm SD & $13.0 \pm 3.5$ & $13.3 \pm 3.4$ & $12.0 \pm 3.7$ & 0.002 \\
\hline Hypertension, n (\%) & $260(45.9)$ & $190(41.5)$ & $70(65.4)$ & $<0.001$ \\
\hline Diabetes, n (\%) & $55(9.7)$ & $41(9.0)$ & $14(13.1)$ & 0.168 \\
\hline Atrial fibrillation, n (\%) & $60(10.6)$ & $40(8.7)$ & $20(18.7)$ & 0.002 \\
\hline Hyperlipidemia, n (\%) & $163(28.8)$ & $130(28.4)$ & $33(30.8)$ & 0.538 \\
\hline Smoking, n (\%) & $159(28.1)$ & $138(30.1)$ & $21(19.6)$ & 0.040 \\
\hline Total Fazekas $\geq 3, \mathrm{n}(\%)$ & $174(30.7)$ & $117(25.5)$ & $57(53.2)$ & $<0.001$ \\
\hline Presence of lacunar infarcts, $n$ (\%) & $77(13.6)$ & $48(10.5)$ & $29(27.4)$ & $<0.001$ \\
\hline Brain volume, ${ }^{a} \mathrm{~cm}^{3}$, mean $\pm S D$ & $1,047.0 \pm 134.0$ & $1,073.8 \pm 129.8$ & $966.9 \pm 115.2$ & $<0.001$ \\
\hline WMH volume, $\mathrm{cm}^{3}$, mean \pm SD & $11.7 \pm 10.9$ & $10.0 \pm 8.9$ & $19.1 \pm 14.7$ & $<0.001$ \\
\hline Average FA (NAWM), mean \pm SD & $0.49 \pm 0.03$ & $0.50 \pm 0.03$ & $0.46 \pm 0.03$ & $<0.001$ \\
\hline Average MD $(\mathrm{NAWM})\left(\times 10^{-3}\right)$, mean \pm SD & $0.90 \pm 0.09$ & $0.88 \pm 0.07$ & $0.99 \pm 0.08$ & $<0.001$ \\
\hline
\end{tabular}

Abbreviations: $F A=$ fractional anisotropy; IQR = interquartile range; $\mathrm{MD}=$ mean diffusivity; NAWM = normal-appearing white matter; $\mathrm{WMH}=$ white matter hyperintensities.

Total Fazekas calculated as sum of deep and periventricular scores.

${ }^{\text {a }}$ Defined as the sum of gray and white matter volumes (i.e., not including the ventricles).

$(F=6.61, p=0.001)$, and a significant interaction between age and cognition $\left(F=4.16, p_{\text {interaction }}=0.016\right)$. The interaction suggested that associations between $\mathrm{WMH}$ and cognitive status are different between the age groups (age $\leq 80$ vs $>80$ years, figure 2). Follow-up one-way ANOVAs confirmed that there were $\mathrm{WMH}$ volume differences across cognitive groups only in patients aged $\leq 80$ and not in patients aged $>80$ (table 2), even when correcting for within-group age $(F=$ $6.17, p=0.002$ for age $\leq 80 ; F=1.06, p=0.351$ for age $>80$ ). Sensitivity analyses showed that the interaction remained significant $\left(p_{\text {interaction }}=0.046\right)$ when adjusting for MRI protocol, sex, years of education, head size, presence of lacunes, and number of vascular risk factors. It also remained significant when repeated using groups of equal sample sizes obtained by randomly splitting the group of young participants in 4 subsets matched on cognition to the original one (table 3).

The association between high WMH load (above vs below the 80th percentile) and dementia (SevereCI vs NoCI) was strong at age $\leq 80$ (odds ratio [OR] 4.0, 95\% confidence interval $[\mathrm{CI}] 1.65-9.71, p=0.001)$ but absent at age $>80$ (OR $0.89,95 \%$ CI $0.26-3.03, p=0.86)$. These associations were unchanged when the analysis was repeated with the 80th percentile cutoff determined separately in the 2 age groups (age $\leq 80$ : OR 3.9, 95\% CI 1.68-9.17, $p=0.001$; age $>80$ : OR $1.6,95 \%$ CI $0.41-6.47, p=0.488)$.

A $2 \times 3$ factorial ANOVA with years of education as dependent variable showed no significant interaction between age and cognitive groups $(p=0.693)$, nor did another ANOVA with brain and ventricles volume as dependent variable $(p=0.159)$.

\section{Age-related associations between MD and FA in NAWM and cognitive status}

A $2 \times 3$ factorial ANOVA on average MD values extracted from NAWM showed a main effect of age $(F=73.58, p<$ $0.001)$, a main effect of cognitive status $(F=14.84, p<0.001)$, and a significant interaction between age and cognitive status $\left(F=3.32, p_{\text {interaction }}=0.037\right)$. Sensitivity analyses showed that the interaction on MD from NAWM remained significant when adjusting for MRI protocol, sex, years of education, head size, presence of lacunes, and number of vascular risks factors $\left(p_{\text {interaction }}=0.048\right)$. A similar $2 \times 3$ factorial ANOVA on average FA values extracted from NAWM showed a main 
Table 2 Clinical and imaging features of cognitive groups by age

\begin{tabular}{|c|c|c|c|c|}
\hline & $\mathrm{No} \mathrm{Cl}$ & Mild Cl & Severe $\mathrm{Cl}$ & $p$ Value \\
\hline \multicolumn{5}{|l|}{ Age $\leq 80$ y } \\
\hline $\mathrm{N}_{\mathrm{WMH}}\left(\mathrm{N}_{\mathrm{DTI}}\right)$ & $338(295)$ & $97(83)$ & $24(20)$ & \\
\hline Age, $y$, mean $\pm S D$ & $61.8 \pm 2.5$ & $62.0 \pm 12.6$ & $70.2 \pm 8.7$ & 0.005 \\
\hline Female, n (\%) & $165(48.8)$ & $45(46.4)$ & $18(75.0)$ & 0.036 \\
\hline Event type, TIA, other minor stroke, n (\%) & $254(75.4)$ & $71(73.2)$ & $11(45.8)$ & 0.007 \\
\hline Hypertension, n (\%) & $131(38.9)$ & $44(45.4)$ & $15(62.5)$ & 0.161 \\
\hline Diabetes, n (\%) & $26(7.7)$ & $7(7.2)$ & $8(33.3)$ & 0.001 \\
\hline Atrial fibrillation, $\mathbf{n}(\%)$ & $26(7.7)$ & $12(12.4)$ & $2(8.3)$ & 0.561 \\
\hline Hyperlipidemia, n (\%) & $93(27.6)$ & $28(28.9)$ & $9(37.5)$ & 0.871 \\
\hline Smoking, n (\%) & $107(31.8)$ & $24(24.7)$ & $7(29.2)$ & 0.547 \\
\hline Presence of lacunar infarcts, $n(\%)$ & $33(9.8)$ & $12(12.6)$ & $3(12.5)$ & 0.691 \\
\hline WMH volume, $\mathrm{cm}^{3}$, mean \pm SD & $9.4 \pm 7.9$ & $10.1 \pm 7.8$ & $18.1 \pm 18.4$ & $<0.001^{\mathrm{a}}$ \\
\hline Average FA (NAWM), mean \pm SD & $0.50 \pm 0.03$ & $0.49 \pm 0.03$ & $0.47 \pm 0.03$ & $<0.001^{\mathrm{a}}$ \\
\hline Average MD (NAWM) $\left(\times 10^{-3}\right)$, mean \pm SD & $0.86 \pm 0.06$ & $0.96 \pm 0.07$ & $0.96 \pm 0.07$ & $<0.001^{\mathrm{a}}$ \\
\hline \multicolumn{5}{|l|}{ Age $>80$ y } \\
\hline $\mathrm{N}_{\mathrm{WMH}}\left(\mathrm{N}_{\mathrm{DTI}}\right)$ & $44(42)$ & $46(42)$ & $17(16)$ & \\
\hline Age, $y$, mean $\pm S D$ & $83.9 \pm 3.3$ & $85.9 \pm 4.2$ & $83.4 \pm 2.5$ & 0.009 \\
\hline Female sex, n (\%) & $22(50.0)$ & $22(47.8)$ & $8(47.1)$ & 0.970 \\
\hline Event type, TIA, other minor stroke, n (\%) & $31(72.1)$ & $30(65.2)$ & $11(64.7)$ & 0.749 \\
\hline Hypertension, n (\%) & $26(59.1)$ & $32(69.6)$ & $12(70.6)$ & 0.730 \\
\hline Diabetes, n (\%) & $3(6.8)$ & $8(17.4)$ & $3(17.6)$ & 0.575 \\
\hline Atrial fibrillation, $\mathbf{n}(\%)$ & $6(13.6)$ & $12(26.1)$ & $2(11.8)$ & 0.512 \\
\hline Hyperlipidemia, n (\%) & $13(29.5)$ & $17(37.0)$ & $3(17.6)$ & 0.637 \\
\hline Smoking, n (\%) & $9(20.5)$ & $9(19.6)$ & $3(17.6)$ & 0.901 \\
\hline Presence of lacunar infarcts, $n(\%)$ & $12(27.9)$ & $15(32.6)$ & $2(11.8)$ & 0.256 \\
\hline WMH volume, $\mathrm{cm}^{3}$, mean \pm SD & $15.9 \pm 10.7$ & $21.3 \pm 14.6$ & $21.4 \pm 21.9$ & $0.166^{\mathrm{a}}$ \\
\hline Average FA (NAWM), mean \pm SD & $0.47 \pm 0.03$ & $0.45 \pm 0.03$ & $0.45 \pm 0.04$ & $0.110^{\mathrm{a}}$ \\
\hline Average $M D(N A W M)\left(\times 10^{-3}\right)$, mean $\pm S D$ & $0.96 \pm 0.07$ & $1.01 \pm 0.09$ & $1.01 \pm 0.10$ & $0.067^{\mathrm{a}}$ \\
\hline
\end{tabular}

Abbreviations: $\mathrm{Cl}$ = cognitive impairment; $\mathrm{FA}=$ fractional anisotropy; $\mathrm{MD}=$ mean diffusivity; $\mathrm{NAWM}$ = normal-appearing white matter; $\mathrm{WMH}=$ white matter hyperintensities.

a Comparisons between cognitive groups that were significant for patients $\leq 80$, but not for patients $>80$.

effect of age $(F=41.74, p<0.001)$, and a main effect of cognitive status $(F=13.67, p<0.001)$, but no interaction $(F=$ $\left.1.52, p_{\text {interaction }}=0.219\right)$.

One-way ANOVAs on average MD and FA values extracted from NAWM confirmed that there were differences across cognitive groups only in patients aged $\leq 80$, but not in patients aged $>80$ (table 2 ). Adding age or $\mathrm{WMH}$ volume as covariates (analyses of covariance) did not change the results (table 4 ).

\section{Localization of WMH relevant to cognitive status}

The voxel-wise $2 \times 3$ factorial ANOVA showed an interaction between cognitive status and age group in an area of the left deep frontal white matter (figure 3, blue-light blue). The voxel-wise correlational analysis performed in patients aged $\leq 80$ showed that in this group the association between the probability of having WMH and lower MoCA score was localized in periventricular frontal and parietal white matter areas bilaterally, more extended on the left hemisphere 
Table 3 Results of factorial analyses of variance (ANOVAs) repeated on 4 random subsamples of participants aged $\leq 80$ stratified according to cognition (i.e., same as the original group of 459)

\begin{tabular}{|c|c|c|c|c|c|c|c|}
\hline \multirow[b]{2}{*}{ Stratified random samples aged $\leq 80$} & \multicolumn{4}{|l|}{$\mathbf{N}$} & \multicolumn{3}{|c|}{$2 \times 3$ factorial ANOVA } \\
\hline & $\mathrm{No} \mathrm{Cl}$ & Mild Cl & Severe $\mathrm{Cl}$ & Total & Mean square & $\boldsymbol{F}$ & $p$ Value \\
\hline Random sample 1 & 84 & 24 & 6 & 114 & 0.370 & 3.098 & 0.047 \\
\hline Random sample 2 & 84 & 24 & 6 & 114 & 0.371 & 3.271 & 0.040 \\
\hline Random sample 3 & 85 & 24 & 6 & 115 & 0.661 & 6.307 & 0.002 \\
\hline Random sample 4 & 85 & 25 & 6 & 116 & 0.438 & 3.559 & 0.030 \\
\hline
\end{tabular}

Abbreviation: $\mathrm{Cl}=$ cognitive impairment.

(figure 3, red-yellow) (see resulting maps on neurovault.org/ collections/2763/). The voxel-wise correlational analysis in patients aged $>80$ instead showed no voxel-wise associations between WMH and MoCA score.

Voxel-wise results did not significantly change when controlling for age within each group.

\section{Discussion}

We found high WMH load in patients with previous TIA or minor stroke aged $>80$, but showed that, in this age group, it was not significantly associated with cognitive impairment. High WMH load was strongly associated with cognition only in patients aged $\leq 80$, who were 4 times more likely to have severe impairment than patients aged $\leq 80$ with low WMH load. The lack of significant association between WMH and cognition in patients aged $>80$ also persisted on voxel-wise analyses of WMH distribution, which are expected to be more sensitive than the simple measure of total WMH volume. Finally, we explored if DTI measures of microstructural integrity in NAWM (i.e., outside WMH) would correlate better with cognitive status, but again found no significant associations at age $>80$.

This loss of association between WMH and cognition at older ages might seem at odds with many previous studies showing significant associations between white matter damage and cognition in the general population or in groups of patients aged $>50,60$, or $65 .{ }^{1-3,7}$ However, 2 studies found no associations between WMH and cognition in communitydwelling elderly and stroke survivors older than $80 .^{23,24}$ Our findings, taken together with these 2 previous studies, have important implications for interpretation of brain imaging at older ages. First, MRI has become the recommended first-line imaging investigation for several neurologic conditions affecting older people, and some WMH are almost universally reported in elderly patients. Our results suggest that high WMH loads in patients aged $>80$ may be considered not excessively concerning by clinicians, patients, or their families. However, the presence of WMH at younger ages should prompt further investigation of possible cognitive impairment. Second, MRI markers of white matter damage have been recommended for use as a proxy of vascular cognitive impairment, ${ }^{11,45,46}$ but interpretation may be more complex in patients aged $>80$.

Since WMH represents late-stage macroscopic damage of the white matter that occurs as a result of small vessel disease, we also studied microstructural markers of white matter integrity associated with interstitial fluid mobility and water content (namely MD and FA) outside the WMH regions in macroscopically normal-appearing white matter. These measures have been argued to be better markers of cognitive decline in patients with symptomatic cerebrovascular disease and to be more sensitive to change. ${ }^{11}$ Yet we did not find a strong association between these measures and cognitive status in patients $>80$, suggesting that our findings are not due to intrinsic limitations of the particular MRI marker adopted. Neuropathologic studies also showed that the association between dementia and postmortem evidence of vascular pathology attenuates in the very old. ${ }^{47}$

The lack of association between white matter abnormality and cognitive impairment in patients $>80$ was mainly driven by patients with substantial white matter disease and normal cognition. Patients aged $\leq 80$ with similar degree of damage instead showed severe cognitive impairment. This finding goes against the hypothesis that a certain threshold of WMH is needed to affect cognition. ${ }^{23}$ It also suggests that the lack of correlation between WMH and cognition in patients aged $>80$ could not simply be due to the fact that patients with severe white matter damage might have already died by the age of 80 , as if this were the case the group aged $>80$ would be expected to have little white matter abnormality. However, WMH may have diverse underlying pathologies, some not affecting cognition or life expectancy, others increasing susceptibility to dementia and death (i.e., only patients whose white matter pathology caused dementia had died by the age of 80). In addition, since we studied all consecutive patients presenting to a TIA/stroke clinic, we cannot exclude the possibility of a sampling bias due to the fact that elderly patients with severe cognitive impairment or dementia may 

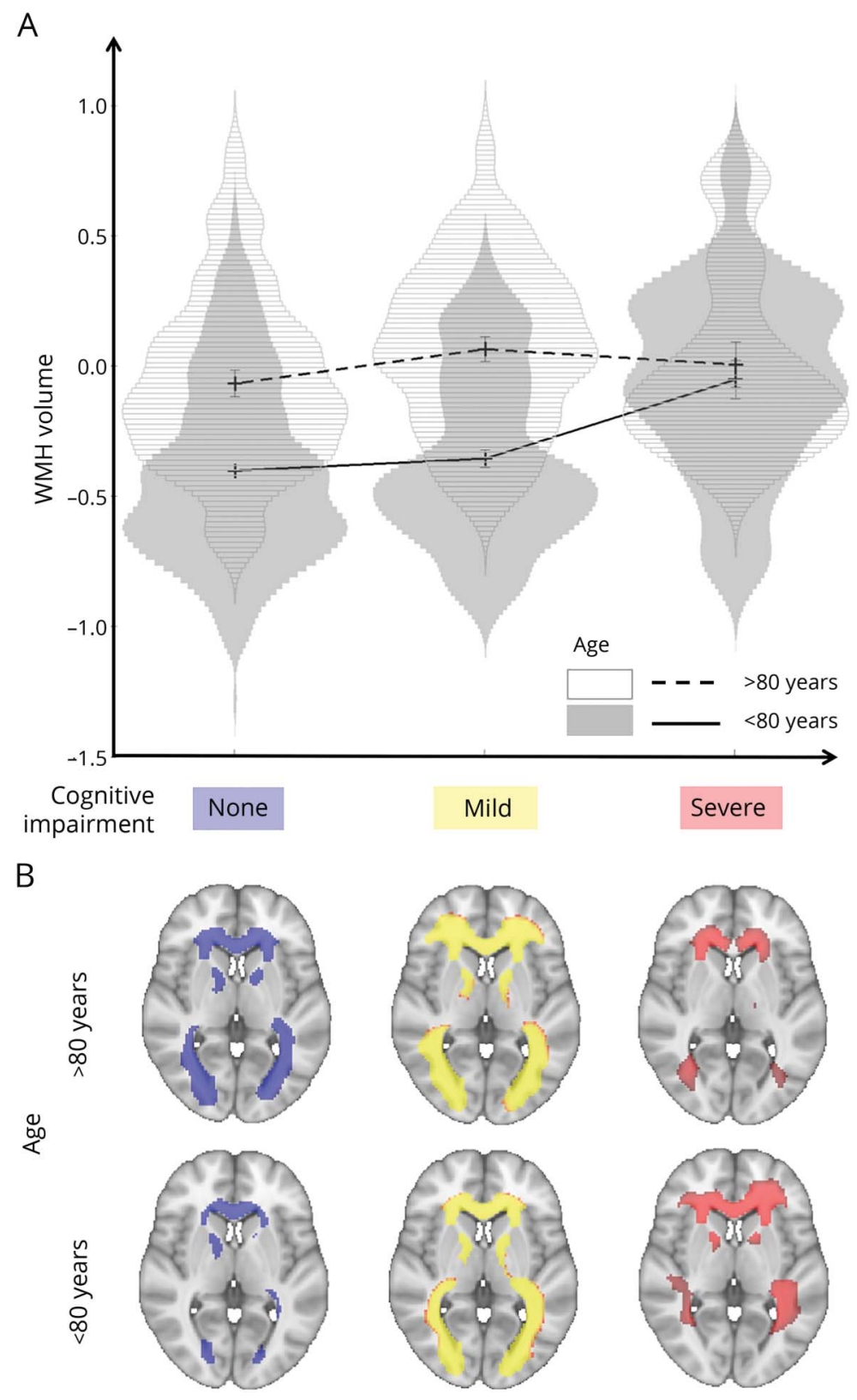

(A) Violin plots of the adjusted, log-transformed WMH volumes for the 6 groups of interest obtained by dividing patients according to cognitive status and age. Error bars are \pm 1 SD. (B) Average maps of WMH distribution for each group. First row, patients aged $>80$; second row, patients $\leq 80$. Left: No cognitive impairment (Cl). Middle: Mild Cl. Right: Severe Cl (neurovault.org/collections/2763/).

not present to medical attention for a suspected TIA. However, previous studies on community-dwelling elderly not subject to presentation bias similarly found no association between white matter abnormalities and cognition. ${ }^{14,23}$ Yet the clinical implications of our study (i.e., the lack of association between $\mathrm{WMH}$ and cognitive impairment in patients aged $>80$ ) remain, irrespective of the mechanism, asultimately - the group of patients relevant to clinicians only includes those who present to medical attention. Of note, the lack of correlation between WMH and cognition in patients aged $>80$ parallels the tendency for the associations between other risk factors and dementia to attenuate with advancing ages. $^{48}$
One of the strengths of our study is that it addresses a major limiting factor in non-population-based studies by not excluding very old patients. In addition, we imaged a relatively homogeneous population in that all patients had recent symptomatic cerebrovascular disease, such that confounding by greater comorbidities or vascular risk factors in the older age group is less likely, as also shown by sensitivity analyses including potential confounders such as brain size, education, number of vascular risks factors, and presence of lacunar infarcts as covariates.

Several limitations should be highlighted. First, we looked only at the cross-sectional associations with cognitive status 
Table 4 Follow-up analyses of covariance performed adjusting for age and for other measures of white matter abnormalities as covariates

\begin{tabular}{|c|c|c|c|c|c|c|c|c|}
\hline \multirow[b]{2}{*}{ Cognitive group effect } & \multicolumn{4}{|l|}{ Age $\leq 80$ y } & \multicolumn{4}{|l|}{ Age $>80$} \\
\hline & $\begin{array}{l}\text { Mean } \\
\text { square }\end{array}$ & $\boldsymbol{F}$ & $\begin{array}{l}p \\
\text { Value }\end{array}$ & $\begin{array}{l}\text { Partial } \\
\eta^{2}\end{array}$ & $\begin{array}{l}\text { Mean } \\
\text { square }\end{array}$ & $\boldsymbol{F}$ & $\begin{array}{l}p \\
\text { Value }\end{array}$ & $\begin{array}{l}\text { Partial } \\
\eta^{2}\end{array}$ \\
\hline $\begin{array}{l}\text { Dependent variable: WMH, no covariates (also reported } \\
\text { in table 2) }\end{array}$ & 1.358 & 11.058 & $<0.001^{a}$ & 0.046 & 0.219 & 2.070 & $0.166^{a}$ & 0.038 \\
\hline Dependent variable: WMH, covariate: age & 0.574 & 6.167 & $0.002^{\mathrm{a}}$ & 0.026 & 0.106 & 1.056 & $0.351^{\mathrm{a}}$ & 0.020 \\
\hline Dependent variable: WMH, covariate: FA in NAWM & 0.312 & 3.987 & $0.019^{a}$ & 0.020 & 0.031 & 0.394 & $0.675^{\mathrm{a}}$ & 0.008 \\
\hline Dependent variable: WMH, covariate: MD in NAWM & 0.113 & 1.584 & 0.206 & 0.008 & 0.013 & 0.196 & 0.822 & 0.004 \\
\hline $\begin{array}{l}\text { Dependent variable: MD, no covariates (also reported in } \\
\text { table 2) }\end{array}$ & $9.578 \mathrm{E}-8$ & 19.487 & $<0.001^{a}$ & 0.090 & $1.985 \mathrm{E}-8$ & 2.782 & $0.067^{a}$ & 0.054 \\
\hline Dependent variable: MD, covariate: age & $4.710 \mathrm{E}-8$ & 14.562 & $<0.001^{\mathrm{a}}$ & 0.069 & $1.426 \mathrm{E}-8$ & 2.234 & $0.113^{a}$ & 0.044 \\
\hline Dependent variable: MD, covariate: WMH & $2.031 \mathrm{E}-8$ & 7.144 & $0.001^{a}$ & 0.035 & 7.015E-9 & 1.568 & $0.214^{a}$ & 0.032 \\
\hline $\begin{array}{l}\text { Dependent variable: FA, no covariates (also reported in } \\
\text { table 2) }\end{array}$ & 0.015 & 18.852 & $<0.001^{a}$ & 0.087 & 0.003 & 2.256 & $0.110^{a}$ & 0.044 \\
\hline Dependent variable: FA, covariate: age & 0.008 & 14.282 & $<0.001^{a}$ & 0.068 & 0.002 & 1.933 & $0.150^{\mathrm{a}}$ & 0.039 \\
\hline Dependent variable: FA, covariate: WMH & 0.005 & 9.053 & $<0.001^{a}$ & 0.044 & 0.001 & 1.262 & $0.288^{a}$ & 0.026 \\
\hline
\end{tabular}

Abbreviations: $\mathrm{FA}$ = fractional anisotropy; $\mathrm{MD}=$ mean diffusivity; $\mathrm{NAWM}=$ normal-appearing white matter; $\mathrm{WMH}$ = white matter hyperintensity.

${ }^{a}$ Analyses of covariance in which the effect of the cognitive groups were significant for patients $\leq 80$, but not for patients $>80$.

and cannot be certain that associations would be similar on long-term follow-up. Second, we used only the MoCA as a screening tool for cognitive impairment, and so we cannot exclude subtler cognitive deficits in older patients with severe white matter disease, which might have been evident on more detailed neuropsychological assessment. However, we were primarily interested in clinically overt cognitive impairment. Third, we did not adjust for the presence of subclinical depression or other neuropsychiatric disorders, which may affect cognitive performance. However, we would expect the effect of stroke-related depression to be similar across the 2 age groups, ${ }^{49}$ as these did not differ in the severity of cerebrovascular event. Finally, we cannot exclude that the lack of correlation between WMH and cognition in patients aged $>80$ was merely due to differences in sample size. However, we believe this possibility to be extremely unlikely considering

Figure 3 Localization of white matter hyperintensity $(\mathrm{WMH})$ relevant to cognitive status
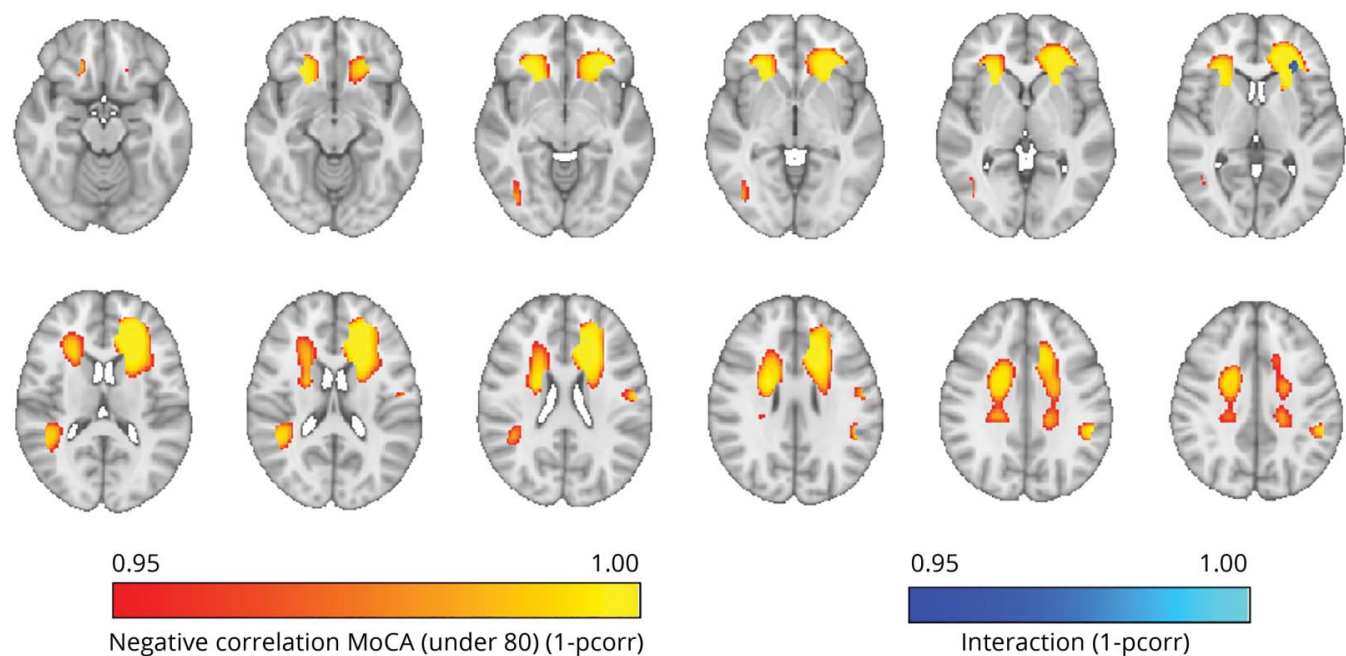

In red-yellow, regions of significant correlation between higher probability of having WMH and lower Montreal Cognitive Assessment scores in patients aged $\leq 80$ years. The same correlational analyses in patients aged $>80$ years did not lead to significant results. In blue-light blue, region of significant interaction between age and cognitive status resulting from the $2 \times 3$ voxel-wise analysis of variance (neurovault.org/collections/2763/). 
that we found a significant interaction and that our results agree with other findings in the literature. ${ }^{14,23,50}$ Future studies with larger sample sizes in patients older than 80 are required to support the identified lack of clinical significance of MRI markers.

Our findings confirm the association between MRI markers of white matter damage and cognitive impairment in patients younger than 80 . They also suggest that the clinical significance of MRI markers might not be overinterpreted in patients older than 80 , or considered a good proxy of vascular cognitive impairment in trials or research studies on this age group.

\section{Author contributions}

G. Zamboni: study design, analysis and interpretation of data, draft and revision of the manuscript. L. Griffanti: data analysis and interpretation, draft and revision of the manuscript. S. Mazzucco: acquisition of data, revision of the manuscript. S.T. Pendlebury: study design and concept, revision of the manuscript. P.M. Rothwell: study design and concept, study supervision and funding, analysis and interpretation of data, critical revision of the manuscript.

\section{Acknowledgment}

The authors thank Dr. Matteo Paolucci and Dr. Riccardo Ricceri for their contribution to quality check of the images. The authors acknowledge the use of the facilities of the Oxford Acute Vascular Imaging Centre (AVIC), Oxford. This work uses data provided by patients and collected by the NHS as part of their care and support and would not have been possible without access to this data. The NIHR recognizes and values the role of patient data, securely accessed and stored, both in underpinning and leading to improvements in research and care.

\section{Study funding}

The Oxford Vascular Study is funded by the Wellcome Trust, Wolfson Foundation, British Heart Foundation, and the NIHR Biomedical Research Centre, Oxford. Professor Pendlebury and Dr. Griffanti were funded by the Oxford NIHR Biomedical Research Centre. The views expressed are those of the authors and not necessarily those of the NHS, the NIHR, or the Department of Health.

\section{Disclosure}

The authors report no disclosures relevant to the manuscript. Go to Neurology.org/N for full disclosures.

\section{Publication history}

Received by Neurology October 25, 2017. Accepted in final form March 4, 2019.

\section{References}

1. Debette S, Markus HS. The clinical importance of white matter hyperintensities on brain magnetic resonance imaging: systematic review and meta-analysis. BMJ 2010; 341:c3666.

2. Mortamais M, Artero S, Ritchie K. Cerebral white matter hyperintensities in the prediction of cognitive decline and incident dementia. Int Rev Psychiatry 2013;25: 686-698.
3. de Groot JC, de Leeuw FE, Oudkerk M, et al. Cerebral white matter lesions and cognitive function: the Rotterdam Scan Study. Ann Neurol 2000;47:145-151.

4. Dong C, Nabizadeh N, Caunca M, et al. Cognitive correlates of white matter lesion load and brain atrophy: the Northern Manhattan Study. Neurology 2015;85: 441-449.

5. Arvanitakis Z, Fleischman DA, Arfanakis K, Leurgans SE, Barnes LL, Bennett DA. Association of white matter hyperintensities and gray matter volume with cognition in older individuals without cognitive impairment. Brain Struct Funct 2016;221:2135-2146.

6. Knopman DS, Griswold ME, Lirette ST, et al. Vascular imaging abnormalities and cognition: mediation by cortical volume in nondemented individuals: Atherosclerosis Risk in Communities: neurocognitive study. Stroke 2015;46:433-440.

7. Biesbroek JM, Kuijf HJ, van der Graaf Y, et al. Association between subcortical vascular lesion location and cognition: a voxel-based and tract-based lesion-symptom mapping study: The SMART-MR study. PLoS One 2013;8:e60541.

8. Zamboni G, Griffanti L, Jenkinson M, et al. White matter imaging correlates of early cognitive impairment detected by the Montreal Cognitive Assessment after transient ischemic attack and minor stroke. Stroke 2017;48:1539-1547.

9. Vernooij MW, Ikram MA, Vrooman HA, et al. White matter microstructural integrity and cognitive function in a general elderly population. Arch Gen Psychiatry 2009;66: 545-553.

10. Tuladhar AM, Reid AT, Shumskaya E, et al. Relationship between white matter hyperintensities, cortical thickness, and cognition. Stroke 2015;46:425-432.

11. Nitkunan A, Barrick TR, Charlton RA, Clark CA, Markus HS. Multimodal MRI in cerebral small vessel disease: its relationship with cognition and sensitivity to change over time. Stroke 2008;39:1999-2005.

12. Maniega SM, Valdés Hernández MC, Clayden JD, et al. White matter hyperintensities and normal-appearing white matter integrity in the aging brain. Neurobiol Aging 2015;36:909-918.

13. Pelletier A, Periot O, Dilharreguy B, et al. Age-related modifications of diffusion tensor imaging parameters and white matter hyperintensities as inter-dependent processes. Front Aging Neurosci 2015;7:255.

14. Bennett IJ, Greenia DE, Maillard P, et al. Age-related white matter integrity differences in oldest-old without dementia. Neurobiol Aging 2017;56:108-114.

15. Munoz Maniega S, Chappell FM, Valdes Hernandez MC, et al. Integrity of normalappearing white matter: influence of age, visible lesion burden and hypertension in patients with small-vessel disease. J Cereb Blood flow Metab 2017;37:644-656.

16. Etherton $\mathrm{MR}, \mathrm{Wu} \mathrm{O}$, Cougo $\mathrm{P}$, et al. Integrity of normal-appearing white matter and functional outcomes after acute ischemic stroke. Neurology 2017;88:1701-1708.

17. de Groot M, Verhaaren BF, de Boer R, et al. Changes in normal-appearing white matter precede development of white matter lesions. Stroke 2013;44:1037-1042.

18. Zhong G, Lou M. Multimodal imaging findings in normal-appearing white matter of leucoaraiosis: a review. Stroke Vasc Neurol 2016;1:59-63.

19. de Leeuw FE, de Groot JC, Achten E, et al. Prevalence of cerebral white matter lesions in elderly people: a population based magnetic resonance imaging study: The Rotterdam Scan Study. J Neurol Neurosurg Psychiatry 2001;70:9-14.

20. Yang Z, Wen W, Jiang J, et al. Age-associated differences on structural brain MRI in nondemented individuals from 71 to 103 years. Neurobiol Aging 2016;40:86-97.

21. Westlye LT, Walhovd KB, Dale AM, et al. Life-span changes of the human brain white matter: diffusion tensor imaging (DTI) and volumetry. Cereb Cortex 2010;20: 2055-2068.

22. Hajjar I, Quach L, Yang F, et al. Hypertension, white matter hyperintensities, and concurrent impairments in mobility, cognition, and mood: the Cardiovascular Health Study. Circulation 2011;123:858-865.

23. Piguet O, Ridley L, Grayson DA, et al. Are MRI white matter lesions clinically significant in the 'old-old'? Evidence from the Sydney Older Persons Study. Demen Geriatr Cogn Disord 2003;15:143-150.

24. Firbank MJ, Burton EJ, Barber R, et al. Medial temporal atrophy rather than white matter hyperintensities predict cognitive decline in stroke survivors. Neurobiol Aging 2007;28:1664-1669.

25. United Nations. Department of International ES, Affairs, Population Division. World population prospects, the 2015 revision. Available at: esa.un.org/unpd/wpp/2015. Accessed June 7, 2019.

26. Yang Z, Slavin MJ, Sachdev PS. Dementia in the oldest old. Nat Rev Neurol 2013;9: 382-393.

27. Lucca U, Tettamanti M, Logroscino G, et al. Prevalence of dementia in the oldest old: the Monzino 80-plus population based study. Alzheimers Dement 2015;11: 258-270.e3.

28. Fotuhi M, Hachinski V, Whitehouse PJ. Changing perspectives regarding late-life dementia. Nat Rev Neurol 2009;5:649-658.

29. Skrobot OA, O'Brien J, Black S, et al. The vascular impairment of cognition classification consensus study. Alzheimers Dement 2017;13:624-633.

30. Jellinger KA. Pathology and pathogenesis of vascular cognitive impairment: a critical update. Front Aging Neurosci 2013;5:17.

31. Love S, Miners JS. White matter hypoperfusion and damage in dementia: postmortem assessment. Brain Pathol 2015;25:99-107.

32. Jagust WJ, Zheng L, Harvey DJ, et al. Neuropathological basis of magnetic resonance images in aging and dementia. Ann Neurol 2008;63:72-80.

33. Lees R, Selvarajah J, Fenton C, et al. Test accuracy of cognitive screening tests for diagnosis of dementia and multidomain cognitive impairment in stroke. Stroke 2014; 45:3008-3018.

34. Pendlebury ST, Mariz J, Bull L, Mehta Z, Rothwell PM. Impact of different operational definitions on mild cognitive impairment rate and MMSE and MoCA performance in transient ischaemic attack and stroke. Cerebrovasc Dis 2013;36:355-362. 
35. Pendlebury ST, Cuthbertson FC, Welch SJ, Mehta Z, Rothwell PM. Underestimation of cognitive impairment by Mini-Mental State Examination versus the Montreal Cognitive Assessment in patients with transient ischemic attack and stroke: a population-based study. Stroke 2010;41:1290-1293.

36. Pendlebury ST, Mariz J, Bull L, Mehta Z, Rothwell PM. MoCA, ACE-R, and MMSE versus the National Institute of Neurological Disorders and Stroke-Canadian stroke network vascular cognitive impairment harmonization standards neuropsychological battery after TIA and stroke. Stroke 2012;43:464-469.

37. Pendlebury ST, Wadling S, Silver LE, Mehta Z, Rothwell PM. Transient cognitive impairment in TIA and minor stroke. Stroke 2011;42:3116-3121.

38. Griffanti L, Zamboni G, Khan A, et al. BIANCA (brain intensity abnormality classification algorithm): a new tool for automated segmentation of white matter hyperintensities. Neuroimage 2016;141:191-205.

39. Smith SM. Fast robust automated brain extraction. Hum Brain Mapp 2002;17: $143-155$.

40. Griffanti L, Jenkinson M, Suri S, et al. Classification and characterization of periventricular and deep white matter hyperintensities on MRI: a study in older adults. NeuroImage 2018;170:174-181.

41. Andersson JLR, Jenkinson M, Smith S. Non-linear optimisation: FMRIB technical report TR07JA1. Available at: www.fmrib.ox.ac.uk/datasets/techrep/tr07ja2/tr07ja2. pdf. Accessed June 7, 2019.

42. Fazekas F, Chawluk JB, Alavi A, Hurtig HI, Zimmerman RA. MR signal abnormalities at 1.5 $\mathrm{T}$ in Alzheimer's dementia and normal aging. AJR Am J Roentgenol 1987;149:351-356.
43. Behrens TE, Woolrich MW, Jenkinson M, et al. Characterization and propagation of uncertainty in diffusion-weighted MR imaging. Magn Reson Med 2003;50: 1077-1088.

44. Winkler AM, Ridgway GR, Webster MA, Smith SM, Nichols TE. Permutation inference for the general linear model. Neuroimage 2014;92:381-397.

45. Wardlaw JM, Smith EE, Biessels GJ, et al. Neuroimaging standards for research into small vessel disease and its contribution to ageing and neurodegeneration. Lancet Neurol 2013;12:822-838.

46. Croall ID, Lohner V, Moynihan B, et al. Using DTI to assess white matter microstructure in cerebral small vessel disease (SVD) in multicentre studies. Clin Sci 2017; 131:1361-1373.

47. Savva GM, Wharton SB, Ince PG, Forster G, Matthews FE, Brayne C. Age, neuropathology, and dementia. N Engl J Med 2009;360:2302-2309.

48. Deckers K, Kohler S, van Boxtel M, et al. Lack of associations between modifiable risk factors and dementia in the very old: findings from the Cambridge City over-75s cohort study. Aging Ment Health 2018;22:1272-1278.

49. Wolfe CD, Crichton SL, Heuschmann PU, et al. Estimates of outcomes up to ten years after stroke: analysis from the prospective South London Stroke Register. PLoS Med 2011;8:e1001033.

50. Firbank MJ, Wiseman RM, Burton EJ, Saxby BK, O'Brien JT, Ford GA. Brain atrophy and white matter hyperintensity change in older adults and relationship to blood pressure: brain atrophy, WMH change and blood pressure. J Neurol 2007;254: $713-721$. 


\section{Neurology}

\section{Age-dependent association of white matter abnormality with cognition after TIA or minor stroke}

Giovanna Zamboni, Ludovica Griffanti, Sara Mazzucco, et al.

Neurology 2019;93;e272-e282 Published Online before print June 14, 2019

DOI 10.1212/WNL.0000000000007772

\section{This information is current as of June 14, 2019}

\section{Updated Information \&} Services

References

Citations

Subspecialty Collections

Permissions \& Licensing

Reprints including high resolution figures, can be found at: http://n.neurology.org/content/93/3/e272.full

This article cites 48 articles, 14 of which you can access for free at: http://n.neurology.org/content/93/3/e272.full\#ref-list-1

This article has been cited by 2 HighWire-hosted articles: http://n.neurology.org/content/93/3/e272.full\#\#otherarticles

This article, along with others on similar topics, appears in the following collection(s):

\section{All Cerebrovascular disease/Stroke}

http://n.neurology.org/cgi/collection/all_cerebrovascular_disease_strok $\mathrm{e}$

\section{Cognitive aging}

http://n.neurology.org/cgi/collection/cognitive_aging

\section{MRI}

http://n.neurology.org/cgi/collection/mri

Vascular dementia

http://n.neurology.org/cgi/collection/vascular_dementia

Information about reproducing this article in parts (figures,tables) or in its entirety can be found online at:

http://www.neurology.org/about/about_the_journal\#permissions

Information about ordering reprints can be found online:

http://n.neurology.org/subscribers/advertise

Neurology ${ }^{\circledR}$ is the official journal of the American Academy of Neurology. Published continuously since 1951, it is now a weekly with 48 issues per year. Copyright Copyright (C) 2019 The Author(s). Published by Wolters Kluwer Health, Inc. on behalf of the American Academy of Neurology.. All rights reserved. Print ISSN: 0028-3878. Online ISSN: 1526-632X.

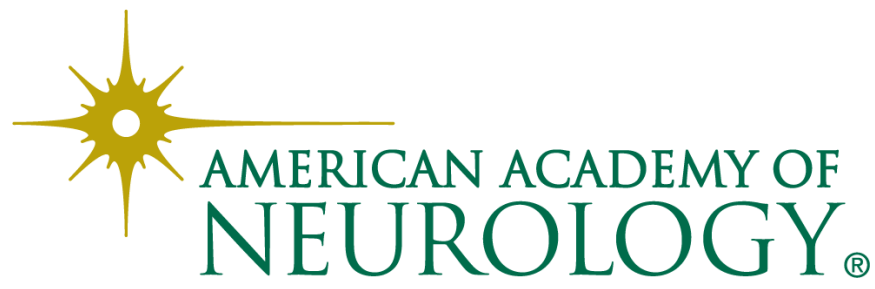

